12. Kupelian PA, Potters L, Khuntia D et al. (2004) Radical prostatectomy, external beam radiotherapy $<72 \mathrm{~Gy}$, external beam radiotherapy >or $=72 \mathrm{~Gy}$, permanent seed implantation, or combined seeds/external beam radiotherapy for stage T1T2 prostate cancer. Int J Radiat Oncol Biol Phys 58: 25-33

13. Lawton CA, Desilvio M, Roach M 3rd et al. (2007) An update of the phase III trial comparing whole pelvic to prostate only radiotherapy and neoadjuvant to adjuvant total androgen suppression: updated analysis of RTOG 94-13, with emphasis on unexpected hormone/radiation interactions. Int J Radiat Oncol Biol Phys 69: 646-655

14. Lawton CA, Winter K, Murray K et al. (2001) Updated results of the phase III Radiation Therapy Oncology Group (RTOG) trial 85-31 evaluating the potential benefit of androgen suppression following standard radiation therapy for unfavorable prognosis carcinoma of the prostate. Int J Radiat Oncol Biol Phys 49: 937-946

15. Mercader M, Bodner BK, Moser MT et al. (2001) T cell infiltration of the prostate induced by androgen withdrawal in patients with prostate cancer. Proc Natl Acad Sci USA 98: 14565-14570

16. Paulson DF, Lin GH, Hinshaw W et al. (1982) Radical surgery versus radiotherapy for adenocarcinoma of the prostate. J Urol 128: 502-504

17. Pilepich MV, Winter K, John MJ et al. (2001) Phase III radiation therapy oncology group (RTOG) trial 86-10 of androgen deprivation adjuvant to definitive radiotherapy in locally advanced carcinoma of the prostate. Int J Radiat Oncol Biol Phys 50: 12431252

18. Pilepich MV, Winter K, Lawton CA et al. (2005) Androgen suppression adjuvant to definitive radiotherapy in prostate carcinoma - long-term results of phase III RTOG 85-31. Int J Radiat Oncol Biol Phys 61: 1285-1290

19. Pollack A, Hanlon AL, Horwitz EM et al. (2004) Prostate cancer radiotherapy dose response: an update of the fox chase experience. J Urol 171: 1132-1136

20. Pollack A, Zagars GK, Starkschall G et al. (2002) Prostate cancer radiation dose response: results of the M. D. Anderson phase III randomized trial. Int J Radiat Oncol Biol Phys 53: 1097-1105

21. Roach M 3rd, Desilvio M, Lawton C et al. (2003) Phase III trial comparing whole-pelvic versus prostate-only radiotherapy and neoadjuvant versus adjuvant combined androgen suppression: Radiation Therapy Oncology Group 9413. J Clin Oncol 21: 1904-1911

22. Roach M 3rd, Marquez C, Yuo HS et al. (1994) Predicting the risk of lymph node involvement using the pre-treatment prostate specific antigen and Gleason score in men with clinically localized prostate cancer. Int J Radiat Oncol Biol Phys 28: 33-37

23. Robinson JW, Moritz S, Fung T (2002) Meta-analysis of rates of erectile function after treatment of localized prostate carcinoma. Int J Radiat Oncol Biol Phys 54: 1063-1068

24. Shipley WU, Lu JD, Pilepich MV et al. (2002) Effect of a short course of neoadjuvant hormonal therapy on the response to subsequent androgen suppression in prostate cancer patients with relapse after radiotherapy: a secondary analysis of the randomized protocol RTOG 86-10. I Intern J Rad Oncol Biol Phys 54: 1302-1310

25. Slater JD, Rossi CJ Jr, Yonemoto LT et al. (2004) Proton therapy for prostate cancer: the initial Loma Linda University experience. Int J Radiat Oncol Biol Phys 59: 348-352
26. Spiotto MT, Hancock SL, King CR (2007) Radiotherapy after prostatectomy: improved biochemical relapse-free survival with whole pelvic compared with prostate bed only for high-risk patients. Int J Radiat Oncol Biol Phys 69: 54-61

27. Tsuji H, Yanagi T, Ishikawa H et al. (2005) Hypofractionated radiotherapy with carbon ion beams for prostate cancer. Int J Radiat Oncol Biol Phys 63: 1153-1160

28. Zelefsky MJ, Cowen D, Fuks Z et al. (1999) Long term tolerance of high dose three-dimensional conformal radiotherapy in patients with localized prostate carcinoma. Cancer 85: 2460-2468

29. Zelefsky MJ, Fuks Z, Hunt M et al. (2001) High dose radiation delivered by intensity modulated conformal radiotherapy improves the outcome of localized prostate cancer. J Urol 166: 876-881

30. Zietman AL, Prince EA, Nakfoor BM et al. (1997) Androgen deprivation and radiation therapy: sequencing studies using the Shionogi in vivo tumor system. Int J Radiat Oncol Biol Phys 38: 1067-1070

31. Zietman AL, Shipley WU (1993) Randomized trials in loco-regionally confined prostate cancer: past, present, and future. Semin Radiat Oncol 3: 10-220
Urologe 2008 • 47:1430

DOI 10.1007/s00120-008-1889-9

Online publiziert: 25. Oktober 2008

(c) Springer Medizin Verlag 2008

\author{
L. Weißbach · C. Schaefer \\ Urologische Klinik, EuromedClinic, Fürth
}

\section{Organ erhaltende Hodentumorchirurgie}

Urologe (2008) 47: 809-817

Der Autor macht darauf aufmerksam, dass im oben genannten Beitrag ein Fehler unterlaufen ist.

Im Kapitel „Operative Organerhaltung bei malignen Hodentumoren " ist die Prozentangabe zur Assoziation einer kontralateralen testikulären intraepithelialen Neoplasie nach Dieckmann et al. [6] falsch. Richtig ist 5,13\% (und nicht 9\% wie angegeben). Die Autoren bitten den Fehler zu entschuldigen.

\section{Korrespondenzadresse \\ Prof. Dr. L. Weißbach \\ Urologische Klinik, EuromedClinic \\ Europa-Allee 1, 90763 Fürth \\ weissbach@euromed.de}

Die Online-Version des Originalartikels können Sie unter http://dx.doi.org/10.1007/s00120-0081711-8 finden. 